\section{MS9-P20 Designed Armadillo repeat proteins serve as scaffolds for the rational assembly of peptide binders with picomolar affinities \\ Peer Mittl ${ }^{1}$, Simon Hansen ${ }^{1}$, Patrick Ernst ${ }^{1}$, Andreas Plückthun ${ }^{1}$}

1. Department of Biochemistry, University of Zürich, Winterthurer Str. 190, 8057 Zürich

email: mittl@bioc.uzh.ch

Background: The specific recognition of macromolecules is key for many applications in biochemical research, medical diagnostics and disease treatment. Currently the development of new recognition molecules depends on the immunization of lab animals or combinatorial biochemistry techniques. Since both approaches are elaborate and require the availability of sufficient amounts of stable target molecules we are developing a modular system that should allow the rational design of sequence-specific peptide binding proteins. This system is based on the armadillo repeat scaffold, because natural armadillo repeat proteins bind their targets in extended anti-parallel conformations with very regular binding topologies.

Results: Although dArmRP with $2^{\text {nd }}$ generation capping repeats were predominantly monomeric in solution, crystal structures of dArmRP with different numbers of internal repeats revealed domain-swapped $\mathrm{N}$-caps. Redesign of the N-cap significantly improved thermodynamic stability and abrogated swapping of N-caps. These $3^{\text {rd }}$ generation dArmRP recognize full-consensus peptides with very high affinities. Dissociation constants decrease exponentially with the number of internal repeats of the dArmRP and the lengths of the target peptides. Several crystal structures of complexes between dArmRPs with five or six internal repeats and full-consensus $(\mathrm{KR})_{5}$ peptides (either free or fused to the $\mathrm{N}$ - and C-terminii of globular proteins) confirm that the binding mode fulfills the expected regular topology. Further crystal structures of complexes between dArmRPs and the mismatch (RR), peptide revealed that $\mathrm{dArmRPs}$ recognize their target peptides in a side-chain specific manner.

Conclusion: Several crystal structures confirm that $3^{\text {rd }}$ generation dArmRPs behave as stable and monomeric molecules that allow the selective recognition of targeted peptides with the expected topology. The logarithmic relationship between dissociation constant and target peptide length suggests a linear relationship between free energy of binding and number of dipeptide-binding modules. Therefore, the rational design of sequence-specific binders with picomolar affinities from a pool of dipeptide-specific armadillo repeats should indeed be possible.

Keywords: protein enginnering, structure/activity relationship, binding proteins, armadillo repeat, solenoid protein
MS10. Structural

bioinformatics (SBI)

Chairs: Guido Capitani, Oliviero Italo Carugo

MS10-P1 Archaeal species evolved

contemporaneously with fungi

William L. Duax ${ }^{1}$

1. Hauptman Woodward MRI

email: duax@ hwi.buffalo.edu

We are currently analyzing the evolution of proteins of the ribosome that have conserved their 3-dimensional fold in all living species for approximately 3 billion years. We have found that although less than $10 \%$ of the residues in aligned ribosomal proteins are conserved at $95 \%$ or greater identity these immutable residues are responsible for the three-dimensional fold of the conserved core of each ribosomal protein. We find that the ribosomal proteins of archaeal species and cytosolic eukaryota share sequence and structural features that distinguish them from those of all bacteria and eukaryotic mitochondria and chloroplasts. This indicates contemporaneous emergence of eukaryota and archaea. This is clearly illustrated in the analysis of ribosomal protein S19 presented here. These studies permit accurate annotation of thousands of gene products that are ambiguously annotated in the TrEMBL database, and indicate that ribosomal proteins can be used to define a rooted evolutionary tree of all cellular species.

Keywords: Evolution, Archaea, Ribosome 